\title{
Research Article \\ Effect of Bed Deformation on Natural Gas Production from Hydrates
}

\author{
Mohamed Iqbal Pallipurath \\ Mechanical Engineering Department, TKM College of Engineering, Kollam, Kerala 691005, India \\ Correspondence should be addressed to Mohamed Iqbal Pallipurath; mohamediqbalp@gmail.com
}

Received 18 December 2012; Accepted 27 March 2013

Academic Editor: Jorge Ancheyta

Copyright ( 2013 Mohamed Iqbal Pallipurath. This is an open access article distributed under the Creative Commons Attribution License, which permits unrestricted use, distribution, and reproduction in any medium, provided the original work is properly cited.

\begin{abstract}
This work is based on modelling studies in an axisymmetric framework. The thermal stimulation of hydrated sediment is taken to occur by a centrally placed heat source. The model includes the hydrate dissociation and its effect on sediment bed deformation and resulting effect on gas production. A finite element package was customized to simulate the gas production from natural gas hydrate by considering the deformation of submarine bed. Three sediment models have been used to simulate gas production. The effect of sediment deformation on gas production by thermal stimulation is studied. Gas production rate is found to increase with an increase in the source temperature. Porosity of the sediment and saturation of the hydrate both have been found to significantly influence the rate of gas production.
\end{abstract}

\section{Introduction}

Energy demand is on the rise globally but the production rates of major fossil fuels are going down. Several analysts predict a drastic reduction in energy production due to diminishing reserve of fossil fuels. The major result from the global analysis is that world oil production peaked in 2006. Production has started to decline at a rate of several percentages per year. This necessitates a search for commercially viable and clean source of energy capable of meeting future energy demands. Natural gas hydrate (NGH) is one of the possible energy sources to meet these requirements. It is a highly condensed form of natural gas formed by capture of natural gas molecules in a cage of water molecules: each cubic meter of natural gas hydrate yields about 160 cubic meter of gas at STP.

A large amount of natural gas hydrate exists on our planet. Such deposits are found both on land (in the permafrost region), and offshore (in the submarine sediment). Over 230 gas hydrate deposits have been found globally. Gas hydrates have also been located in the coastal regions of India [1]. Needless to say, the vastness of gas hydrates has attracted global attention for its exploration and exploitation for future energy supply. It is predicted that utilization of even $17 \%$ to
$20 \%$ of this resource could meet the energy demands for next 200 years [2].

Methods suggested for the production of natural gas from gas hydrate include depressurization, thermal stimulation, chemical inhibitor injection, and $\mathrm{CO}_{2}$ sequestration. Among these, depressurization and thermal stimulation have been considered to be the most economical, though other methods are under investigation. The type of method depends on the reservoir characteristics. Due to less energy input for depressurization, this method has been studied more than the thermal stimulation. However, the efficacy of latter method needs to be studied in more detail. In recent times, during December 2001 to March 2002, field tests were conducted at a permafrost region located at Mallik gas hydrate site (Canada) in which both depressurization and thermal stimulation were conducted. Each of these methods has its relative merits and demerits, and for the development of commercial technology it is essential to have a good understanding of each of the proposed methods.

\section{Current Scenario}

The study of any of the methods for gas production from natural gas hydrates needs not only a proper knowledge of 
the thermodynamics, kinetics, and heat and mass transfer effects, but also that of sediment response to any change in its morphology during hydrate dissociation. The presence of hydrate has a cementing effect on the sediment structure and hence any loss of hydrate tends to weaken the sediment. Sediment deformation has profound influence on the gas productivity. Moreover, a reduction in the sediment strength may destabilize sediment matrix to an extent that could cause serious damage to the production operation and uncontrolled release of methane to the environment.

The earlier modeling on gas production from gas hydrates considered an undeformed bed as the main objective was to study the effectiveness of a given method in the gas production. Some of these works considered the kinetics of hydrate dissociation (e.g., [3]), and others assumed instantaneous dissociation and hence equilibrium condition during hydrate dissociation (e.g., [4]). Also, the heat transfer effect was not considered in all the studies (e.g., [3] assumed isothermal operation). Analytical solutions were obtained by Selim and Sloan [4], Yousif et al. [3], Tsypkin [5], Ji et al. [6], and many others. However, such solutions were based on assumptions which were found not to be valid in reality. Numerical approach to the problem has been attempted to address the real conditions existing in the reservoir and other operational issues. Gas production from hydrate reservoir by the combination of warm water flooding and depressurization was proposed by Bai and Li [7] which can overcome the deficiency of single production method. Sun et al. [8] developed a onedimensional model of hydrate depressurization in porous media.

Recently, a research group led by Professor Kimoto [9, 10] from Kyoto University, Japan, reported studies on gas production from gas hydrates considering bed deformation. They proposed a model based on chemothermomechanically coupled analysis which could predict the deformation of sediment; they did not report the gas production under this condition. Gas production from gas hydrate was also studied by some researchers by using some of the reservoir simulators such as CMG-STARS, Hydrate Res Sim, MH-21 HYDRES, STOMP-HYD, and TOUGH-HYDRATE.

Kinetics and thermodynamics of hydrate formation and dissociation dictate the choice of operating conditions and hence the gas production. Pioneering work on methane hydrate kinetics was done by Professor Bishnoi and his research team from University of Calgary (e.g., [11-13]). Other studies on methane hydrate kinetics were performed with different additives such as polymeric inhibitor [14], electrolyte solution [15], and promoter [16] A kinetic rate model was proposed by Kim et al. [13] for methane hydrate decomposition. Kinetics of hydrate formation of gases other than $\mathrm{CH}_{4}$, such as $\mathrm{C}_{2} \mathrm{H}_{6}$, and natural Gas, was studied by Kaschiev and Firoozabadi [17].

The gas hydrate thermodynamics dictates the pressuretemperature relationship to predict the zone of hydrate stability. Also, the heat of dissociation of hydrate is obtained from such studies. Selim and Sloan [4] reported a thermodynamic relationship based on Antoine equation and also an equation to determine the heat of hydrate dissociation. Lu and Sultan (2008) summarized the studies on hydrate stability and proposed a correlation to relate pressure and temperature at hydrate equilibrium which gave a good match with the previous experimental data.

The effects of some polymers and surfactants on methane hydrate formation were investigated by Karaaslan and Parlaktuna (2004) in a high-pressure system.

Results of field test on gas production by thermal stimulation of hydrated sediments performed at Mallik 5L-38 gas hydrate production research well were reported by Hancock et al. [18]. No other field data on thermal stimulation is available.

A review of the reported studies in the literature shows that the following issues need to be investigated in more detail.

(i) The type of soil mechanical model that can best predict the production behaviour during thermal stimulation has not previously been investigated. The previous work available in the literature on the effect of soil deformation indicates that soil model may have a significant bearing on the gas production.

(ii) Till now, experimental data are available only from one field test on thermal stimulation in permafrost region. It is necessary to conduct experiments to study this method under submarine conditions.

2.1. Description of the Model Used. Numerical modelling can be an effective tool that enables understanding mechanisms leading to wellbore instability in oceanic hydrate bearing sediment. To assess deformations caused by hydrate dissociation and the effect of these deformations on the gas generation, numerical techniques are essential. Thermal dissociation of hydrated sediment by a pumped hot fluid is modeled. A radial heat flow from the hot pipe is assumed. The coordinate system is cylindrical. Four components (soil, hydrate, gas (methane), and water) and three phases (hydrate, gas, and aqueousphase) are considered in the simulator. The intrinsic kinetics of hydrate formation or dissociation is considered using the Kim-Bishnoi model. Mass transport and heat transfer involved in formation or dissociation of hydrates are included in the governing equations. The arrangement of heat source is shown in Figures 1 and 2.

Factors to be evaluated are as follows.

(i) Changing the stresses and pore pressures.

(ii) Impact of the selected constitutive model for pore pressures.

(iii) Effect of heating the formation on thermodynamic stability of the hydrates.

(iv) Deformation of the sediment as a result of dissociation of hydrate.

(v) Effect of this deformation on the gas production rates.

(vi) Effect of different saturations of hydrate on gas production.

(vii) Effect of source temperature on gas production.

(viii) Effect of porosity on gas production. 
Mechanisms to be considered are as follows.

(i) Kinetic rate, heat and mass transfer equilibrium, and fluid flow relations for gas hydrate dissociation/reformation with the change in pressure and temperature.

(ii) Resultant changes in the mechanical and petrophysical properties of the sediments.

(iii) Representative constitutive equation and yield criterion for the mechanical behavior of HBS of various hydrate concentrations.

(iv) Different soil models and their responses to deformation.

The generation rates of gas and water are dictated by stoichiometry of the hydrate. The transition between gas, water, and hydrates can be represented as a chemical reaction

$$
g\left(\frac{G}{A}\right)+n \cdot w(A) \Longleftrightarrow h(H),
$$

where $g$ is the gas component, existing as free gas $(G)$ or dissolved in water $(A), h$ is the hydrate component present only in the hydrate phase $(H)$, and $n$ is the hydration number.

\subsection{Assumptions}

(i) Gas hydrate bearing zone at a total depth of 3000 to 3300 meters ( $\sim 700 \mathrm{~m}$ below sea floor and $2400 \mathrm{~m}$ below sea level) is considered the default range of study (Yun et al., 2010).

(ii) We neglect the adsorption of any component by the rock phase; that is, the rock phase is inactive in mass transfer.

(iii) Momentum (fluid flow) and heat transfer are axisymmetric.

(iv) The water phase is incompressible.

(v) The gas follows the Peng Robinson equation of state.

(vi) The porous medium (rock) is nondeformed.

(vii) Gas can occur only in gaseous and hydrate states since $\mathrm{CH}_{4}$ solubility under conditions of model is negligible.

(viii) Water can occur only in liquid and hydrate states; that is, ice and water vapour formation are neglected since the sediment conditions preclude its formation.

Equivalent thermal conductivity of hydrated sediment is given by the equation

$$
\lambda=(1-\varphi) \lambda_{s}+\sum_{j=h, g, w} \phi S_{j} \lambda_{j} .
$$

For a porosity of 0.47 , let us consider two extreme cases of hydrate saturation, namely, 0.8 and 0.1 . For hydrate saturation of 0.8 and water and gas saturations of 0.1 each, the equivalent thermal conductivity is $3.968 \mathrm{~W} \mathrm{~m}^{-1} \mathrm{~K}^{-1}$ whereas for a hydrate saturation of 0.1 and water and gas saturations of 0.45 each, the equivalent thermal conductivity is $3.868 \mathrm{~W} \mathrm{~m}^{-1} \mathrm{~K}^{-1}$ which is a difference of just $2.53 \%$. This shows that an assumption of invariant equivalent thermal conductivity is valid.

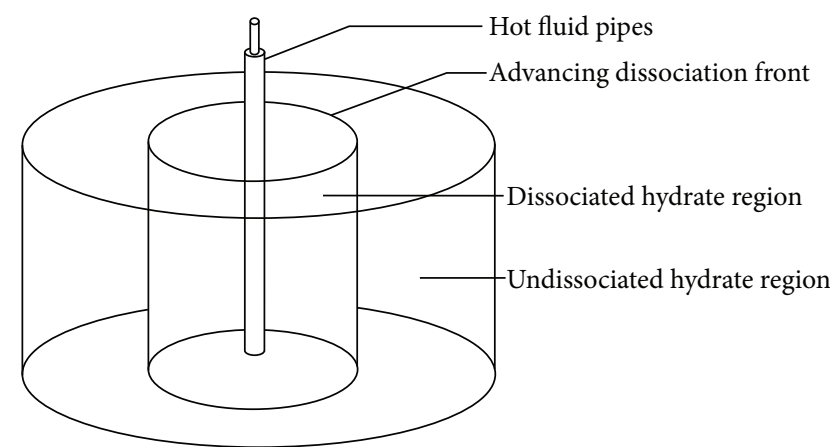

FIGURE 1: Schematic of a hydrate reservoir heated with pipes carrying hot fluid.

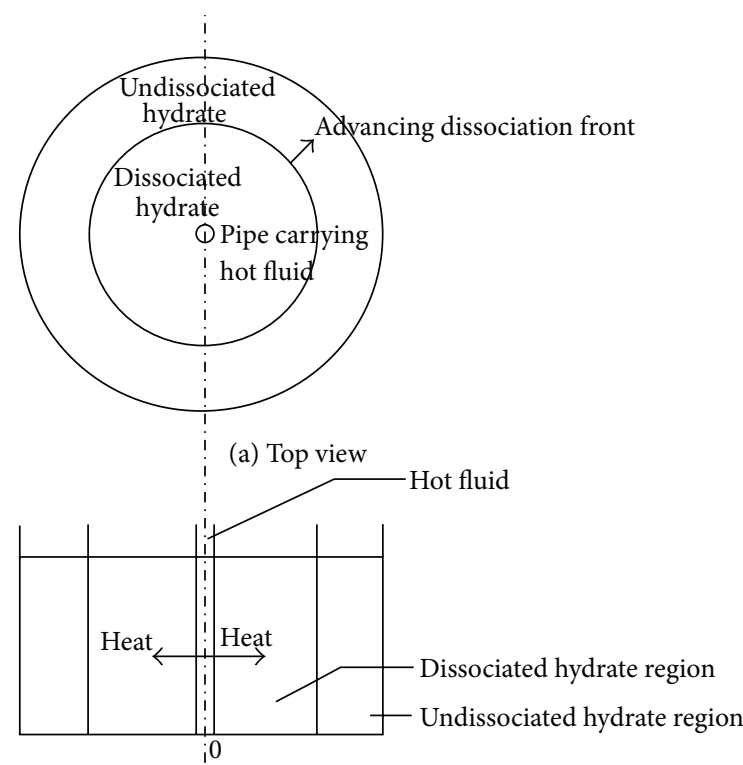

Dissociation front

(b) Side view

FIGURE 2: Schematic representation of hydrated sediment to study gas hydrate dissociation by thermal stimulation.

2.3. Flow through Porous Media Applied to Hydrate Bearing Sediment. A porous medium is modelled in Abaqus/Standard by a conventional approach that considers the medium as a multiphase material and adopts an effective stress principle to describe its behaviour. The porous medium modelling provided considers the presence of two fluids in the medium. One is the "wetting liquid," which is assumed to be relatively (but not entirely) incompressible. Often the other is a gas, which is relatively compressible. An example of such a system is marine hydrated sediment containing sea water and gas. When the medium is partially saturated, both fluids exist at a point; when it is fully saturated, the voids are completely filled with the wetting liquid.

The porous medium is modelled by attaching the finite element mesh to the solid phase; fluid can flow through this mesh. 
2.4. Coupled Flow and Heat Transfer through Porous Media. Optionally, heat transfer due to conduction in the soil skeleton and pore fluid, as well as convection in the pore fluid, can also be modeled. This capability represents an enhancement to the basic pore fluid flow capabilities discussed in the earlier paragraphs and requires the use of coupled temperature-pore pressure elements that have temperature as an additional degree of freedom in addition to the pore pressure and the displacement components. When you use the coupled temperature-pore pressure elements, Abaqus solves the heat transfer equation in addition to and in a fully coupled manner with the continuity equation and the mechanical equilibrium equations. Only linear brick, first-order axisymmetric, and second-order modified tetrahedrons are available for modeling coupled heat transfer with pore fluid flow and mechanical deformation. Coupled temperature-pore pressure elements are not supported in Abaqus/CAE.

2.5. Total and Excess Pore Fluid Pressure. The coupled pore fluid diffusion/stress analysis capability can provide solutions either in terms of total or "excess" pore fluid pressure. The excess pore fluid pressure at a point is the pore fluid pressure in excess of the hydrostatic pressure required to support the weight of pore fluid above the elevation of the material point. The difference between total and excess pore pressure is relevant only for cases in which gravitational loading is important. Total pore pressure solutions are provided when the gravity distributed load is used to define the gravity load on the model. Excess pore pressure solutions are provided in all other cases, for example, when gravity loading is defined with body force distributed loads.

2.6. Transient Analysis. In this transient coupled pore pressure/effective stress analysis, the backward difference operator is used to integrate the continuity equation and the heat transfer equation: this operator provides unconditional stability so that the only concern with respect to time integration is accuracy.

For fully saturated flow analyses in which heat transfer is also modelled, the contributions to the model's stiffness matrix arising from convective heat transfer due to pore fluid flow are unsymmetric.

2.7. Partially Saturated Flow. In gas hydrate sediment analysis, we shall be dealing with partially saturated flow. In partially saturated flow cases, the corresponding guideline for the minimum time increment is

$$
\Delta t>\frac{\gamma_{w} n^{0}\left(1+\beta v_{w}\right)}{6 k_{s} k} \frac{d s}{d u_{w}}(\Delta \ell)^{2},
$$

where $s$ is the saturation, $k_{s}$ is the permeability-saturation relationship, $d s / d u_{w}$ is the rate of change of saturation with respect to pore pressure, $n^{0}$ is the initial porosity of the material, $\Delta t$ is the time increment, $\gamma_{w}$ is the specific weight of the wetting liquid, $k$ is the permeability of the soil, $v_{w}$ is the magnitude of the velocity of the pore fluid, $\beta$ is the velocity coefficient in Forchheimer's flow law $(\beta=0)$ in the case of Darcy flow, and $\Delta \ell$ is a typical element dimension.
2.8. Automatic Incrementation. Since automatic time incrementation is left to Abaqus, three tolerance parameters are chosen. The accuracy of the time integration of the flow continuity equations is governed by the maximum wetting liquid pore pressure change, $\Delta u_{w}^{\max }$, allowed in an increment. Abaqus/Standard restricts the time increments to ensure that this value is not exceeded at any node (except nodes with boundary conditions) during any increment in the analysis.

Since heat transfer is modelled, the accuracy of time integration is also governed by the maximum temperature change, $\Delta \theta_{\text {max }}$, allowed in an increment. Abaqus/Standard restricts the time increments to ensure that this value is not exceeded at any node (except nodes with boundary conditions) during any increment of the analysis.

2.9. Mechanical Constitutive Models. The constitutive library provided in Abaqus contains a range of linear and nonlinear material models for all of these categories of materials. In general, the library has been developed to provide those models that are most usually required for practical applications. There are several distinct models in the library, and for the more commonly encountered materials, several ways of modeling the material are provided, each suitable to a particular type of analysis application. But the library is far from comprehensive: the range of physical material behavior is far too broad for this ever to be possible. If there is no model in the library that is useful for a particular case, Abaqus/ Standard contains a user subroutine UMAT. In these routines the user can code a material model (or call other routines that perform that task). This "user subroutine" capability proved to be a powerful resource for the modelling of gas hydrate bearing sediment.

From a numerical viewpoint, the implementation of a constitutive model involves the integration of the state of the material at an integration point over a time increment during a nonlinear analysis (the implementation of constitutive models in Abaqus assumes that the material behavior is entirely defined by local effects, so each spatial integration point can be treated independently). Since Abaqus/Standard is most commonly used with implicit time integration, the implementation must also provide an accurate "material stiffness matrix" for use in forming the Jacobian of the nonlinear equilibrium equations.

\subsection{FEM Formulation of Gas Production from Sediment Bed.} The Abaqus package was customized with user subroutines and modified input files to solve the model equations. Some typical results obtained for submarine sediment are given. Figure 3 is a comparison of the three developed soil models for the base case using simulation of cumulative gas production for comparison. The three models of soil dynamical system give comparable results as shown in Figure 3.

In Figures 4 and 5, the temperature profiles for the viscoplastic and viscoelastic soil models are compared, and the figures indicate that, by around 60 hours, the temperature profile steadies and is unvarying with time thereafter. It may be inferred that, for a given radius of reservoir, there exists 


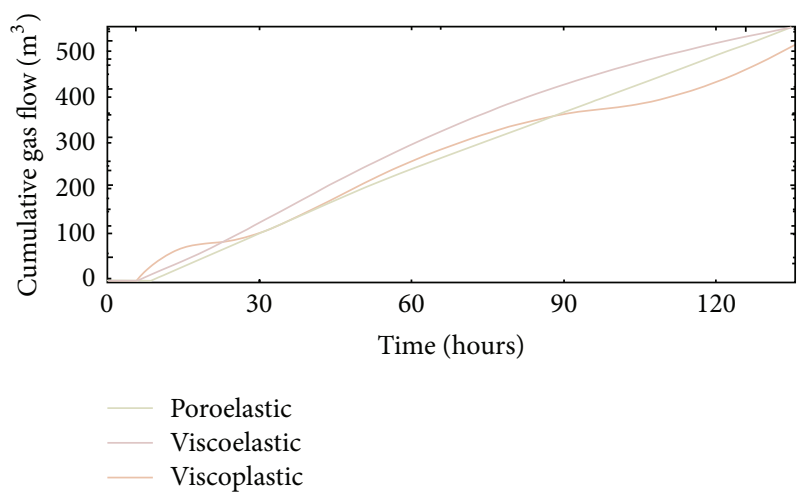

FIGURE 3: Three soil models compared in cumulative gas production rate.

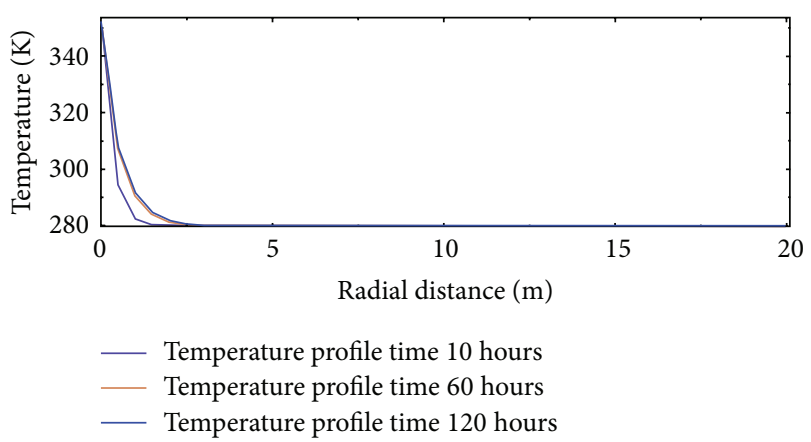

FIGURE 4: Temperature profile in sediment at various times using viscoplastic model.

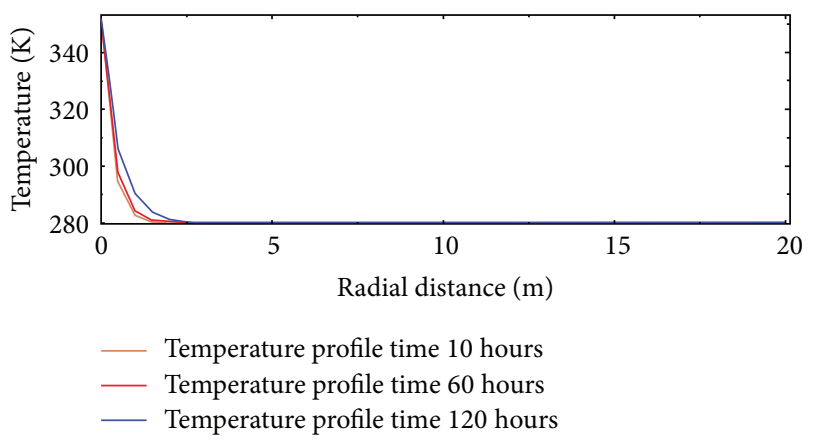

FIgURE 5: Temperature profile in sediment at various times using viscoelastic model.

a constant period of time after which the temperature profile can be said to have achieved steady state.

It appears from the curves in Figures 4 and 5 that the steady state temperature distribution is not dependant on the soil model, provided the thermal properties of the sediment are more or less constant, as they are in these two soil models. But, as can be seen, the rates at which the steady states are achieved can vary marginally with the soil models used.

The effects of deformation on gas production are shown in Figures 6 and 7. Figure 6 shows cumulative gas production without deformation. When the plots are compared side by

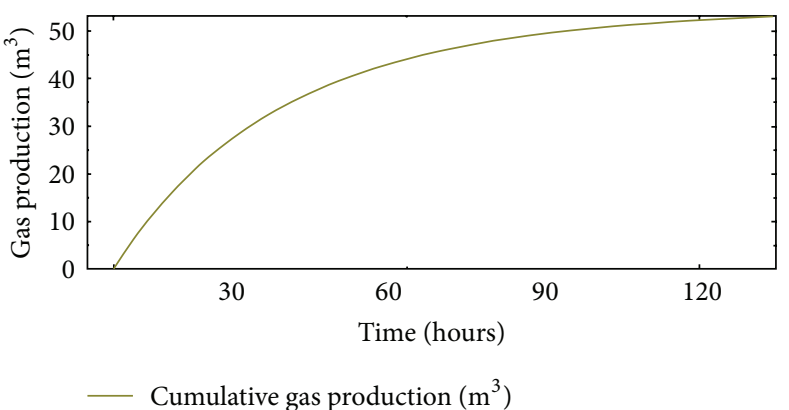

FIgURE 6: Cumulative gas production without deformation.

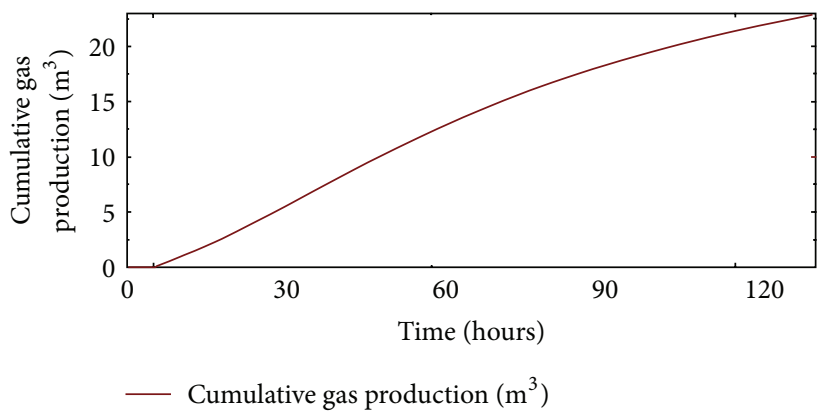

FIGURE 7: Cumulative gas production with deformation (viscoelastic soil model).

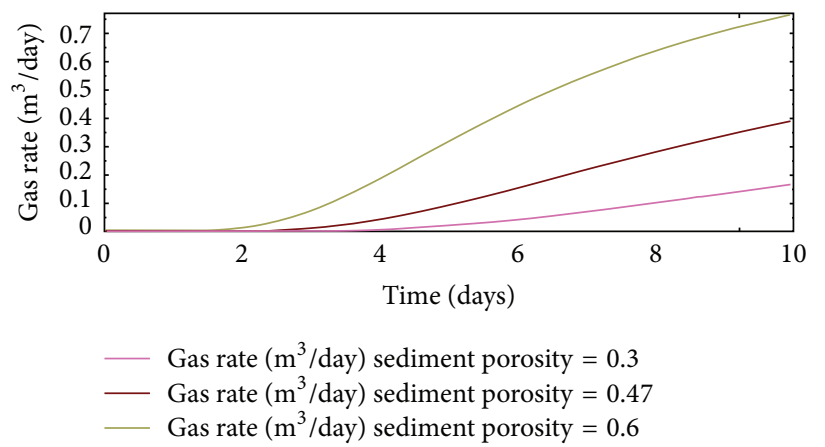

FIGURE 8: Effect of initial bed porosity on gas production rate.

side, it appears that the amount of gas produced is reduced by more than $50 \%$ when we allow for deformation of the sediment. This could be explained by the reduced permeability of the sediment after dissociation and subsequent subsidence.

Figure 7 shows cumulative gas production with deformation of sediment bed using the viscoelastic soil model. The gaps between the sediment grains are reduced after subsidence, which could account for the drop in gas/water permeability, which in turn reduces the gas production by more than half.

2.11. Parametric Study. A few representative results are shown of parametric studies conducted to gauge the impact of porosity and hydrate saturation upon gas production rate. The effect of sediment porosity upon gas production is graphically presented in Figure 8. Higher porosity seems to aid 


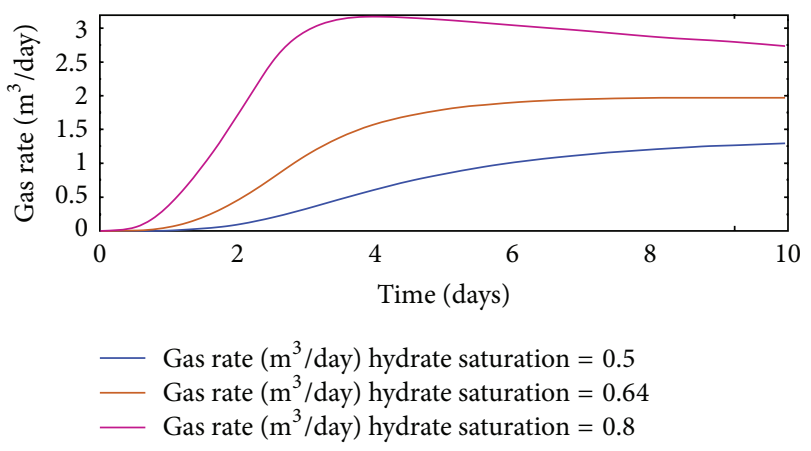

FIGURE 9: Effects of initial hydrate saturation on gas production rate.

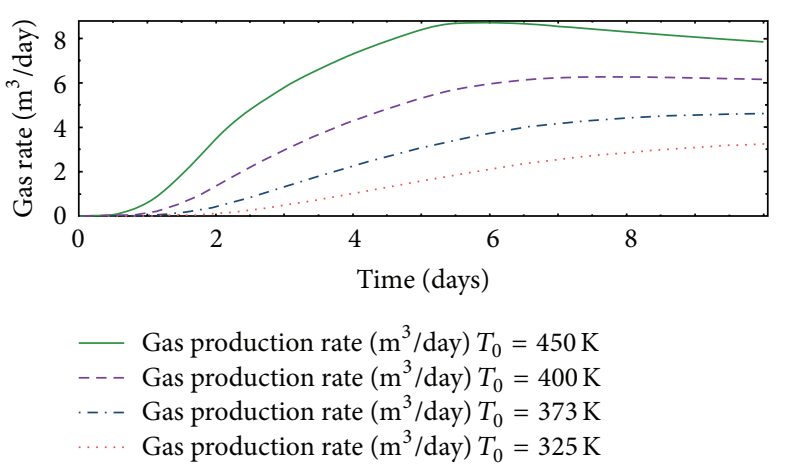

FIgURE 10: Effect of heat source temperature upon the gas production rate.

the production rate due to greater mobility for gas in higher porosity sediment (with higher permeability).

Gas production rates at different initial hydrate saturations are shown in Figure 9. The production rates are dependent on initial hydrate saturation in a way that is expected, with higher saturations yielding greater amount of gas produced per day.

For a constant temperature of the heat source, a higher saturation of hydrate is expected to correspond to larger volumes of produced $\mathrm{CH}_{4}$ because of larger hydrate abundance. The substantial increase in the volume of the released gas when hydrate saturation increases from 0.5 to 0.8 as shown in Figure 9 confirms this expectation. Higher saturations of hydrate mean richer sediment and higher production rates. The effect of gas production rate on the heat source temperature is shown below in Figure 10.

The heat source temperature has a marked influence upon the rate of production of gas, with a factor of $~ 50 \%$ increase in production with $12.5 \%$ increase in temperature. But maintaining the heat source temperature at say $450 \mathrm{~K}$ is a costly proposition. A more economically sustainable temperature would be around 350-360 K.

2.12. Validation of Model. The three soil models were validated with the well data from JAPEX/JNOC/GSC and others, Mallik 5L-38, gas hydrate production research well [18] as shown in Figures 11 and 12.

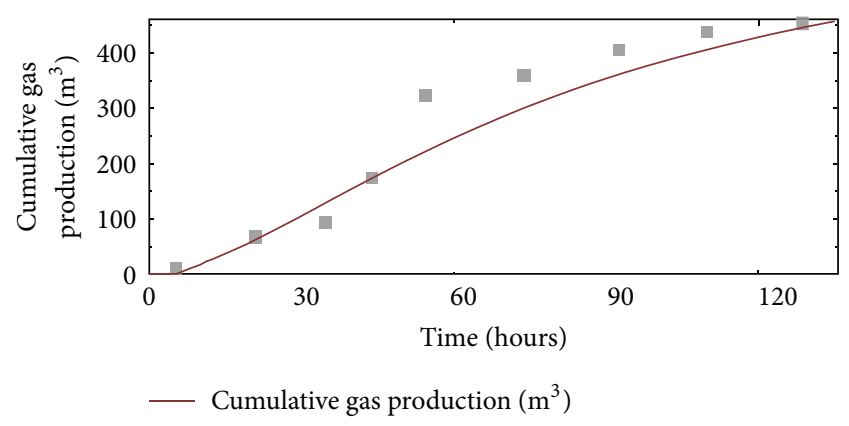

FIGURE 11: Poroelastic model of sediment validated with Mallik 5L38 well data.

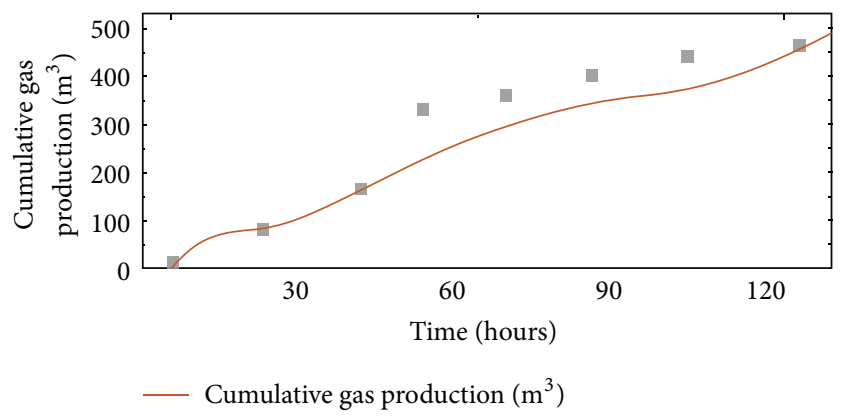

FIGURE 12: Viscoplastic model of sediment validated with Mallik 5L38 well data.

2.13. FEM Profiles. Figures $13,14,15,16$, and 17 show the raw Abaqus screen output for parameters such as nodal temperature, temperature, and porosity across the width and depth of sediment.

\section{Conclusions}

A commercial package Abaqus was customized to simulate the gas production from natural gas hydrate by considering the deformation of submarine bed. The effect of sediment deformation on gas production by thermal stimulation is studied. The effect of three soil dynamic models, which are generally used for submarine sediments, on the prediction of the gas production has been studied. Gas production rate is found to increase with an increase in the source temperature. Porosity of the sediment and saturation of the hydrate both have been found to significantly influence the rate of gas production.

The economics of the method of gas production from gas hydrates using thermal stimulation has been evaluated in the literature prior to this work. But such evaluations (Table 1) have always assumed that the gas production rate cannot be increased significantly by raising the temperature of the heating fluid. This study has found evidence to the contrary (Figure 10) which, though not conclusive, opens up new possibilities in the economic extraction of natural gas from ocean gas hydrate deposits worldwide in general and the $\mathrm{KG}$ and $\mathrm{KK}$ basins in India in particular. 
TABLE 1: Economics of gas production from hydrates (modified from [19]).

\begin{tabular}{lccc}
\hline & & Gas extraction methods \\
& Thermal stimulation & Depressurization & Conventional gas production \\
\hline Investment in millions of ₹* & 254200 & 166000 & 157500 \\
Annual cost in millions of ₹ & 160000 & 125500 & 100000 \\
Total production (million $\mathrm{m}^{3} /$ year) & 1274.26 & 1557.43 & 1557.43 \\
Production cost $\left(₹ /\right.$ million $\mathrm{m}^{3}$ ) & 6356.6 & 4025.9 & 3213.6 \\
Break-even wellhead price $\left(₹ /\right.$ million $\mathrm{m}^{3}$ ) & 7945.8 & 5032.3 & 3972.9 \\
\hline
\end{tabular}

${ }^{*}$ Calculated for currency rates at 2004 levels.

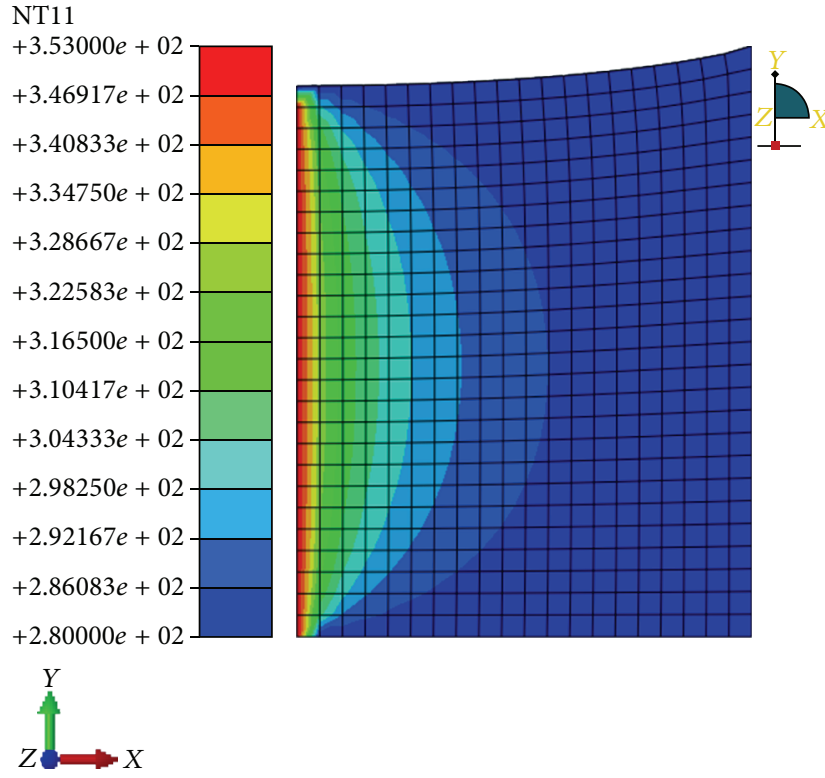

FigURE 13: Nodal temperature profile of the sediment bed (with deformation). Radius of bed is $10 \mathrm{~m}$, and depth is $13 \mathrm{~m}$. Initial temperature was $280 \mathrm{~K}$.

The higher overall annual average temperature of the regions where these basins are located do mitigate to some extent the energy overhead required to maintain a high temperature flow to the sediment bed.

The earlier techniques of hot fluid injection have many limitations as compared to the method studied in this work. In the earlier method, the heat losses to adjacent sediments are too big to permit economic extraction of gas from hydrate reservoirs by steam injection, even if the steam can be introduced at high rates $(15 \mathrm{MW})$ into impenetrable hydrate reservoirs.

So also, low injection temperatures involve very big volumetric flow rates to carry useful amounts of heat into the reservoir. Injection of approximately $3600 \mathrm{~m}^{3}$ per day of $66^{\circ} \mathrm{C}$ water is required if a heat flux of $15 \mathrm{MW}$ is to be maintained. The limitations of disproportionate heat losses on the one hand and unrealistically high injection flow rates on the other hand will probably limit injection temperatures to between 66 and $120^{\circ} \mathrm{C}$.

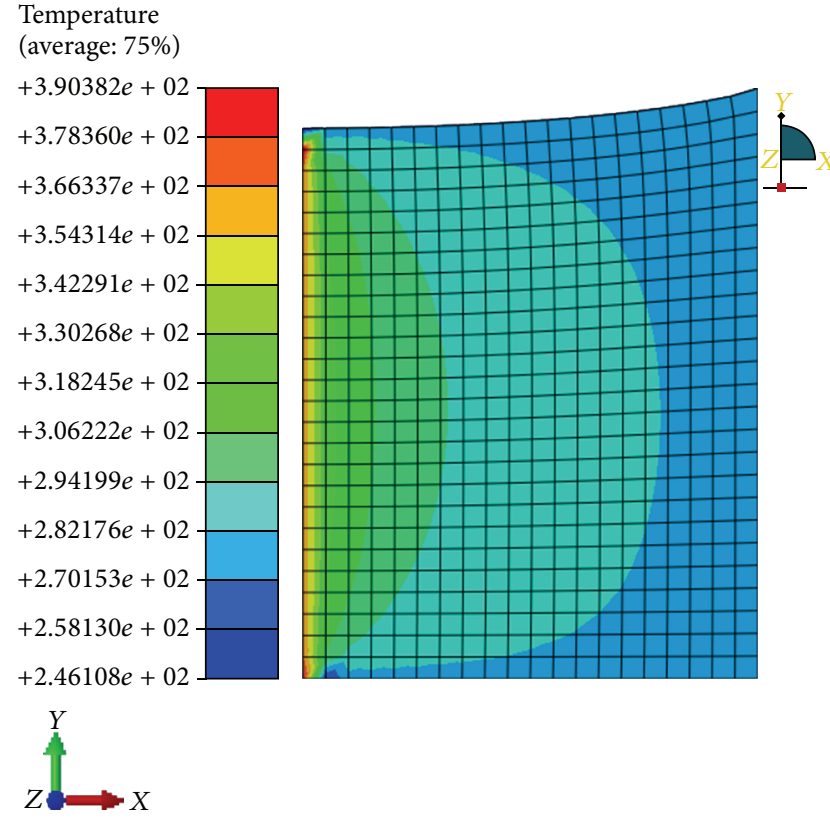

FIgURE 14: Temperature distribution after 50 days (with deformation).

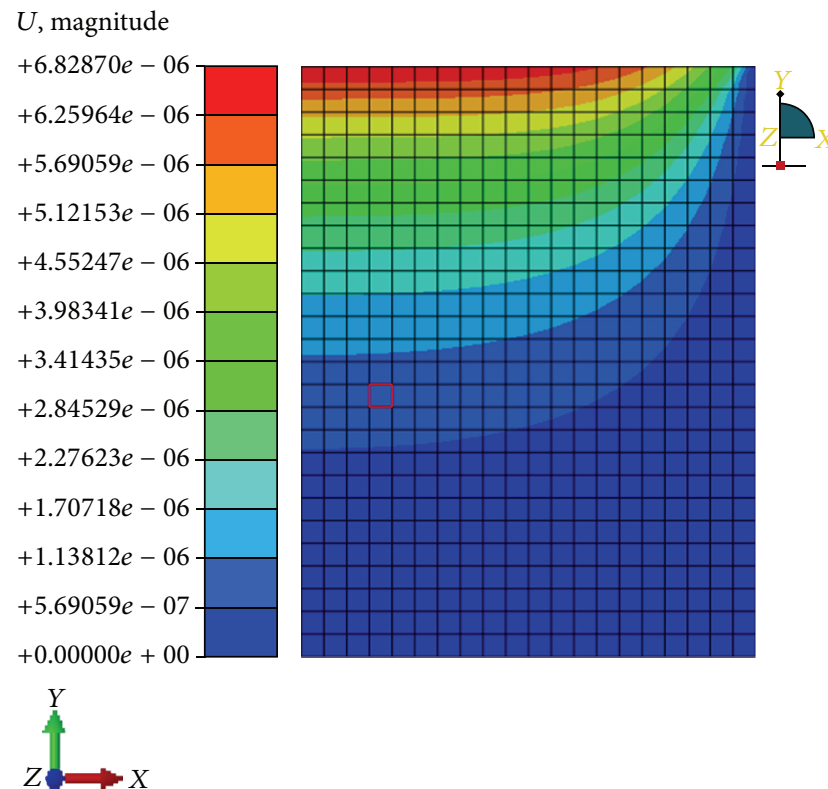

FIGURE 15: Magnitude of deformation (in $\mathrm{m}$ ) after 6 hours. 


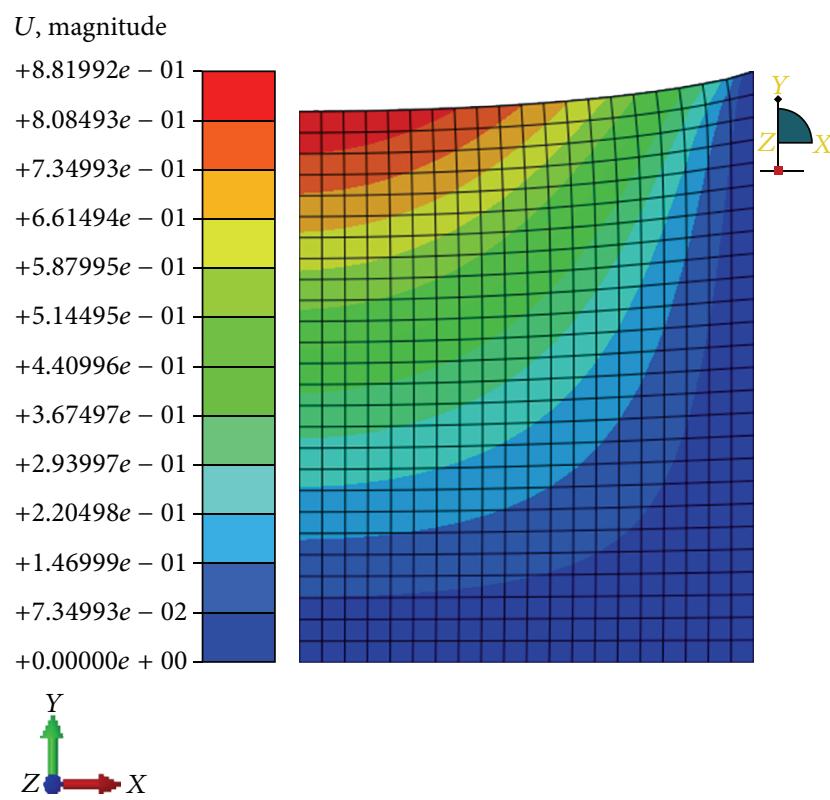

FIgURE 16: Magnitude of deformation after 50 days (m).

\section{UVARM7}

(average: $75 \%$ )
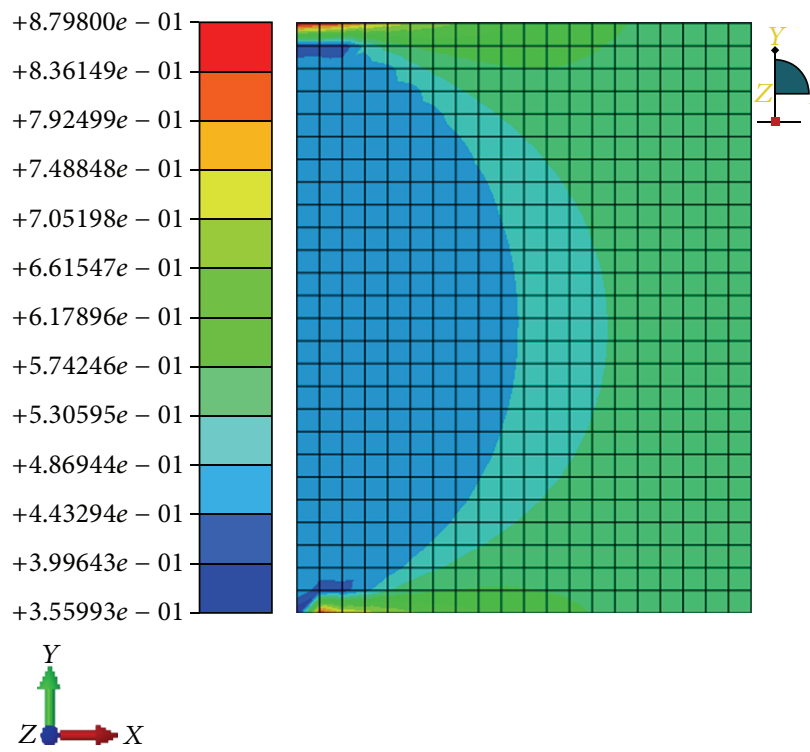

FIgURE 17: Porosity of sediment after 50 days.

Similarly, unless the porosity is at least 0.15 , the heat lost in raising the soil matrix temperature will render thermal stimulation (by injection) ineffective in producing useful quantities of gas. The relative importance of porosity in determining gas production is illustrated in Figure 8.

Scope for future work could be as follows.

(i) Consideration of inhomogeneity and anisotropy of the hydrate bed.

(ii) Study of the interaction between the multiphase flow and the porous bed. (iii) Study of hybrid and alternate techniques for gas production.

(iv) Further experimental validation of the above simulated results.

\section{References}

[1] “DGH Annual Activity Report 2010-2011," Hydrocarbon exploration and production activities.

[2] Y. F. Makogon, "Natural gas hydrates: a promising source of energy," Journal of Natural Gas Science and Engineering, vol. 2, no. 1, pp. 49-59, 2010.

[3] M. H. Yousif, H. H. Abass, M. S. Selim, and E. D. Sloan, "Experimental and theoretical investigation of methane gas hydrate dissociation in porous media," in Proceedings of the SPE Annual Technical Conference \& Exhibition, pp. 571-18320, October 1988.

[4] M. S. Selim and E. D. Sloan, "Heat and mass transfer during the dissociation of hydrate in porous media," AIChE Journal, vol. 35, pp. 1049-1052, 1989.

[5] G. G. Tsypkin, "Mathematical model for dissociation of gas hydrates coexisting with gas in strata," Doklady Physics, vol. 46, no. 11, pp. 806-809, 2001.

[6] C. Ji, G. Ahmadi, and D. H. Smith, "Natural gas production from hydrate decomposition by depressurization," Chemical Engineering Science, vol. 56, no. 20, pp. 5801-5814, 2001.

[7] Y. Bai and Q. Li, "Simulation of gas production from hydrate reservoir by the combination of warm water flooding and depressurization," Science China Technological Sciences, vol. 53, no. 9, pp. 2469-2476, 2010.

[8] X. Sun, N. Nanchary, and K. K. Mohanty, "1-D modeling of hydrate depressurization in porous media," Transport in Porous Media, vol. 58, no. 3, pp. 315-338, 2005.

[9] S. Kimoto, F. Oka, T. Fushita, and M. Fujiwaki, "A chemothermo-mechanically coupled numerical simulation of the subsurface ground deformations due to methane hydrate dissociation," Computers and Geotechnics, vol. 34, no. 4, pp. 216-228, 2007.

[10] S. Kimoto, F. Oka, and T. Fushita, "A chemo-thermo-mechanically coupled analysis of ground deformation induced by gas hydrate dissociation," International Journal of Mechanical Sciences, vol. 52, no. 2, pp. 365-376, 2010.

[11] A. Vysniauskas and P. R. Bishnoi, "A kinetic study of methane hydrate formation," Chemical Engineering Science, vol. 38, no. 7, pp. 1061-1072, 1983.

[12] P. Englezos, N. Kalogerakis, P. D. Dholabhai, and P. R. Bishnoi, "Kinetics of formation of methane and ethane gas hydrates," Chemical Engineering Science, vol. 42, no. 11, pp. 2647-2658, 1987.

[13] H. C. Kim, P. R. Bishnoi, R. A. Heidemann, and S. S. H. Rizvi, "Kinetics of methane hydrate decomposition," Chemical Engineering Science, vol. 42, no. 7, pp. 1645-1653, 1987.

[14] J. S. Pic, J. M. Herri, and M. Cournil, "Experimental influence of kinetic inhibitors on methane hydrate particle size distribution during batch crystallization in water," Canadian Journal of Chemical Engineering, vol. 79, no. 3, pp. 374-383, 2001.

[15] P. D. Dholabhai, N. Kalogerakis, and P. R. Bishnoi, "Kinetics of methane hydrate formation in aqueous electrolyte solutions," Canadian Journal of Chemical Engineering, vol. 71, no. 1, pp. 6874, 1993. 
[16] N. Gnanendran and R. Amin, "Modelling hydrate formation kinetics of a hydrate promoter-water-natural gas system in a semi-batch spray reactor," Chemical Engineering Science, vol. 59, no. 18, pp. 3849-3863, 2004.

[17] D. Kashchiev and A. Firoozabadi, "Driving force for crystallization of gas hydrates," Journal of Crystal Growth, vol. 241, no. 1-2, pp. 220-230, 2002.

[18] S. H. Hancock, T. S. Collett, and S. R. Dallimore, "Overview of thermal-stimulation production test results for the JAPEX/ JNOC/GSC et al. Mallik 5L-38 gas hydrate production research well," 2005.

[19] B. S. Pierce and T. S. Collett, "Energy resource potential of natural gas hydrates," in Proceedings of the 5th Conference Exposition on Petroleum Geophysics, pp. 899-903, Hyderabad, India, 2004. 

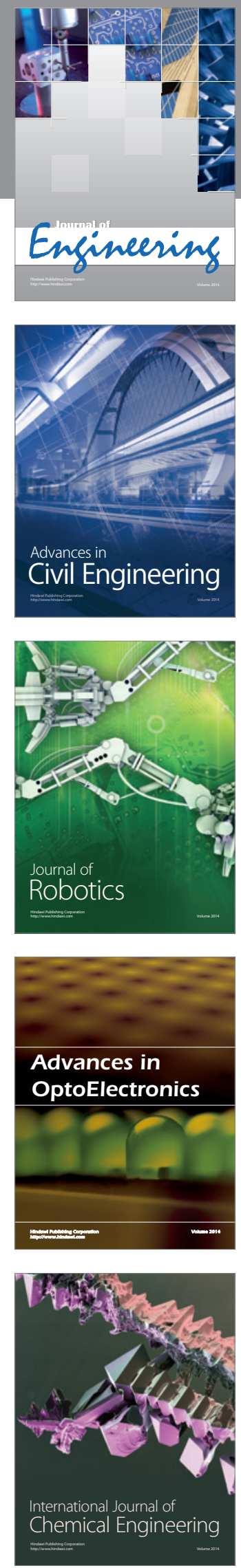

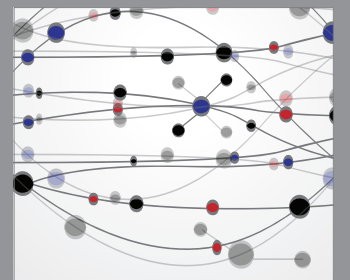

The Scientific World Journal
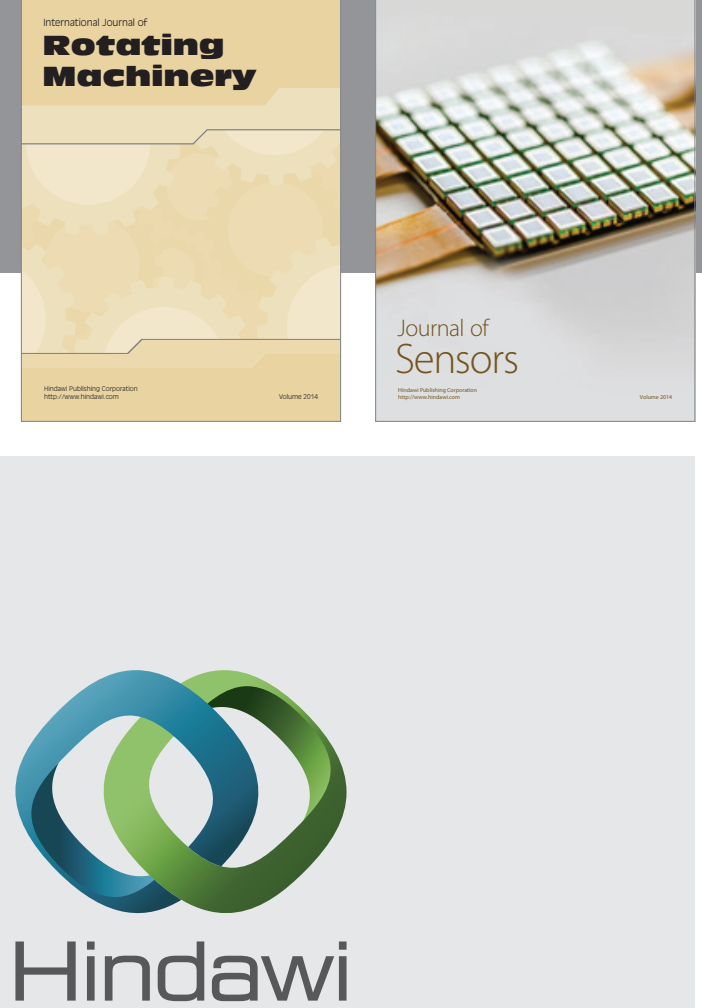

Submit your manuscripts at http://www.hindawi.com
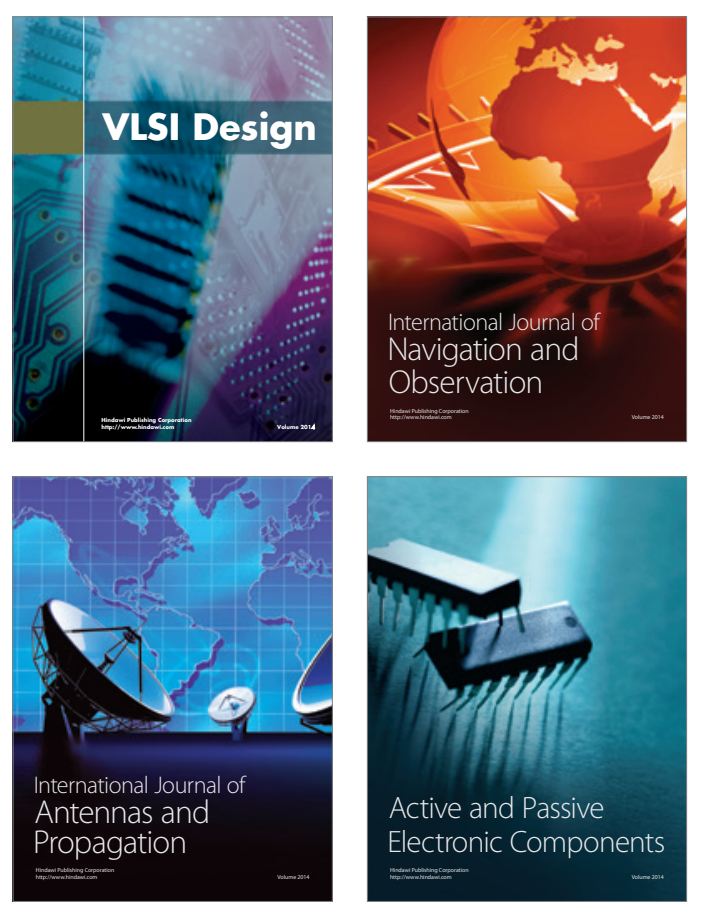
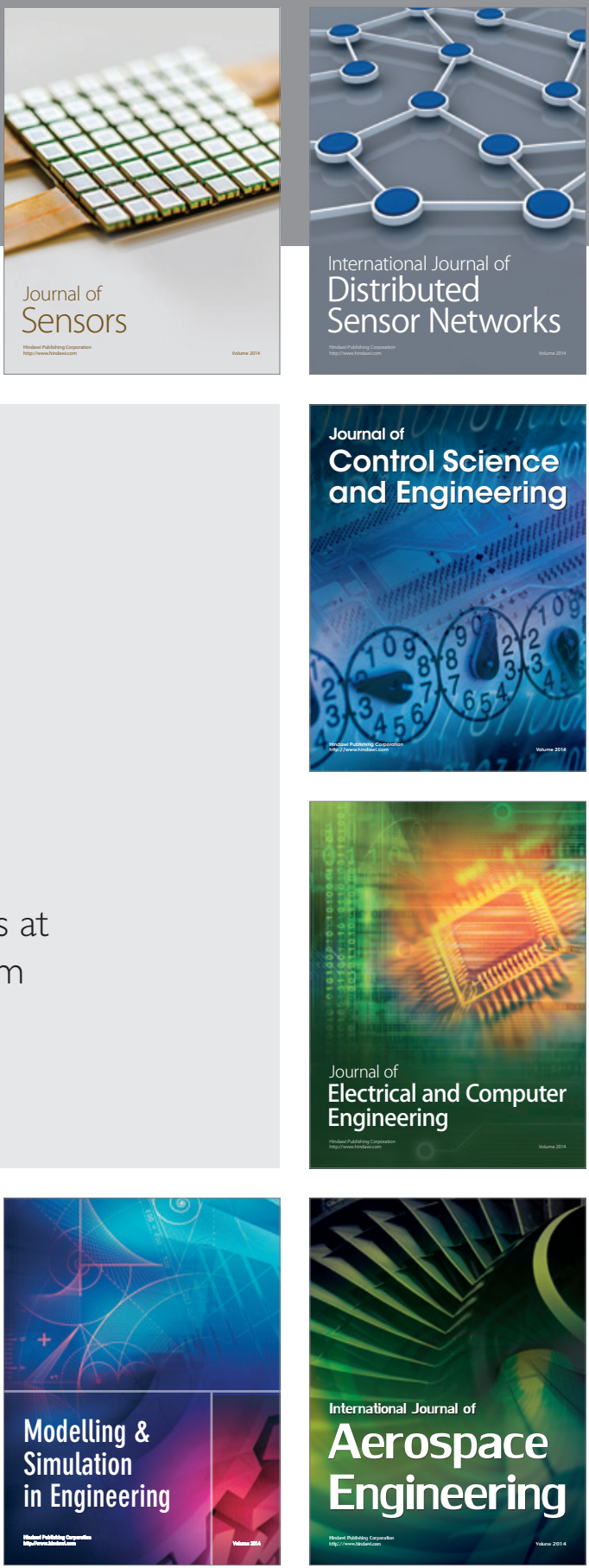

Journal of

Control Science

and Engineering
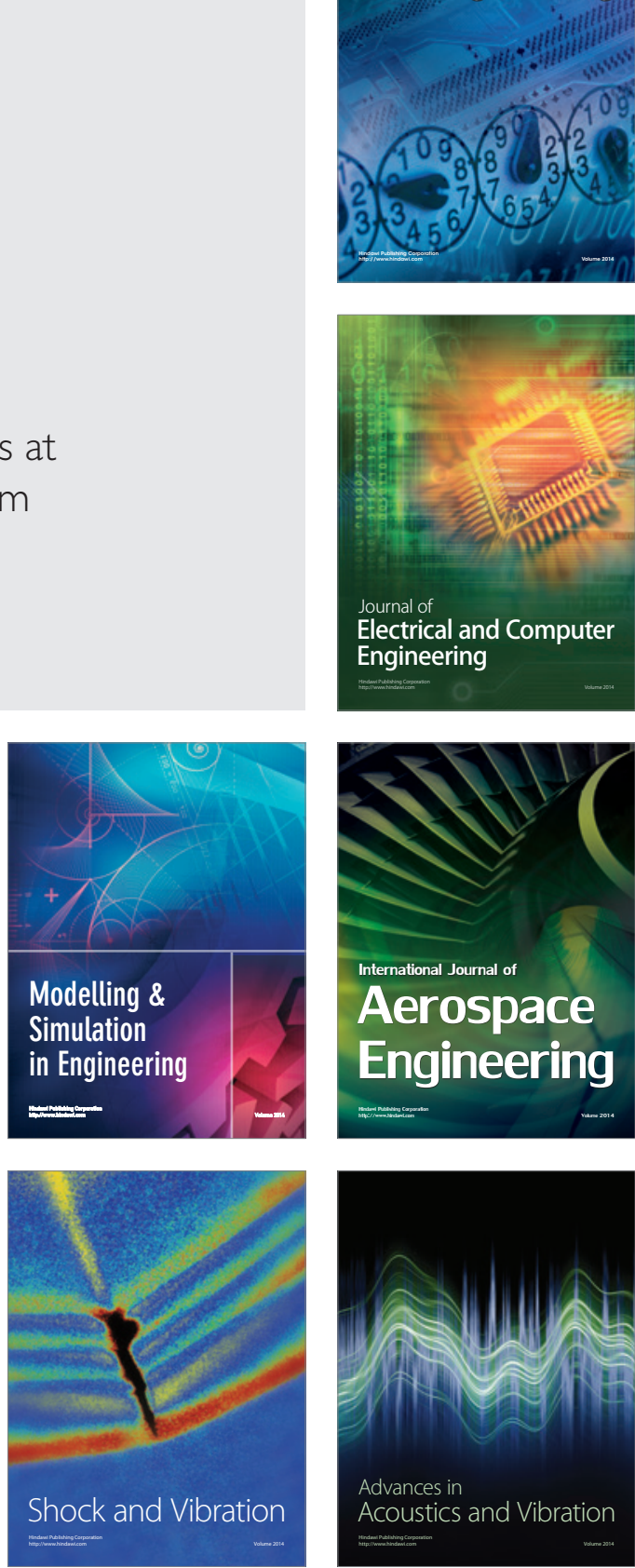